\title{
Combination Therapy in Renal Cell Carcinoma: the Best Choice for Every Patient?
}

\author{
Ernesto Rossi ${ }^{1} \cdot$ Melissa Bersanelli ${ }^{2} \cdot$ Alain Jonathan Gelibter $^{3} \cdot$ Nicolò Borsellino $^{4} \cdot$ Claudia Caserta $^{5} \cdot$ Laura Doni $^{6}$. \\ Marco Maruzzo ${ }^{7} \cdot$ Alessandra Mosca $^{8} \cdot$ Carmela Pisano $^{9} \cdot$ Elena Verzoni $^{10} \cdot$ Paolo Andrea Zucali $^{11,12}$
}

Accepted: 6 July 2021 / Published online: 8 November 2021

(c) Springer Science+Business Media, LLC, part of Springer Nature 2021

\begin{abstract}
Purpose of Review Therapeutic alternatives to treat metastatic renal cell carcinoma (mRCC) are increasing, and combination therapies, including antiangiogenic agents and tyrosine kinase/mTOR/immune checkpoint inhibitors, are identified as the gold standard driven by the results of recent clinical studies. Nevertheless, the real-world RCC population is very heterogeneous, with categories of patients not represented in the enrolled trial population who may not benefit more from these treatments. The purpose of this expert review is to assess the rationale on which tyrosine kinase alone may still be a viable first-line treatment option for some subgroups of patients with mRCC.

Recent Findings The first-line treatment with tyrosine kinase inhibitor monotherapy can still be considered an effective tool for addressing selected mRCCs, as highlighted by the successful outcome in a range of subjects such as favorable-risk patients, the ones suffering from autoimmune diseases, those with pancreatic or lung metastases, or previously undergoing organ transplantation and elderly subjects.

Summary Some selected categories of patients may still benefit from monotherapy with TKI, and smart sequential therapies can also be considered instead of a combination strategy. Tyrosine kinase inhibitors can also act as immune modulator agents, boosting the immune response to facilitate and potentiate the therapeutic effectiveness of subsequent immunotherapy.
\end{abstract}

Keywords $\mathrm{mRCC} \cdot$ Combination therapy $\cdot$ Antiangiogenic factors $\cdot$ Tyrosine kinase inhibitors (TKI) Immune checkpoint inhibitors (ICI) $\cdot$ Immunotherapy

This article is part of the Topical collection on Genitourinary

Cancers

Ernesto Rossi

ernestorossi.rm@gmail.com;

ernesto.rossi@policlinicogemelli.it

1 Medical Oncology, Fondazione Policlinico Universitario Agostino Gemelli IRCCS, Rome, Italy

2 Medicine and Surgery Department, University of Parma and Medical Oncology Unit, University Hospital of Parma, Parma, Italy

3 Medical Oncology Unit B, "Sapienza" University of Rome, Policlinico Umberto I, Rome, Italy

4 Medical Oncology, Buccheri La Ferla - Fatebenefratelli Hospital, Palermo, Italy

5 Medical and Translational Oncology, Azienda Ospedaliera Santa Maria, Terni, Italy
6 Medical Oncology, Azienda Ospedaliero Universitaria Careggi, Firenze, Italy

7 Medical Oncology Unit 1, Department of Oncology, Istituto Oncologico Veneto IOV IRCCS, Padova, Italy

8 Multidisciplinary Outpatient Oncology Clinic, Candiolo Cancer Institute, FPO-IRCCS, Candiolo, Turin, Italy

9 Department of Urology and Gynecology, Istituto Nazionale Tumori IRCCS Fondazione G. Pascale, Napoli, Italy

10 Medical Oncology, Fondazione IRCCS Istituto Dei Tumori, Milan, Italy

11 Department of Biomedical Sciences, Humanitas University, Pieve Emanuele, Milan, Italy

12 Department of Oncology, IRCCS Humanitas Research Hospital, Rozzano, Milan, Italy 


\section{Introduction}

The therapeutic armamentarium for the treatment of metastatic renal cell carcinoma (mRCC) is increasingly rich nowadays. The definition of a therapeutic algorithm is a crucial issue, considering the complexity of the factors involved in the progression of the disease and the heterogeneity of the population affected. Because of the lack of validated predictive factors, the choice of treatment is made even more difficult. Furthermore, the characteristics of "real life" patients may be different from those enrolled in clinical trials.

The current treatments for mRCC include systemic therapies such as several tyrosine kinase inhibitors (TKIs) or immune checkpoint inhibitors (ICIs) $[1 \bullet \bullet, 2]$. Such therapies can be administered sequentially, but, more recently, combination therapies have been introduced with the aim to improve patient outcomes. These therapies act synergistically on different targets and pathways involved in tumor progression. As suggested by the promising results of recent clinical trials, combination therapies are believed to be of greater importance in the treatment of $\mathrm{RCC}[3 \bullet$, $4 \bullet, 5 \bullet \bullet, 6,7 \bullet]$. While combined therapies are becoming the standard of treatment, it is not entirely clear whether such approaches are the best option for all patients. Indeed, the purpose of this expert review is to assess the rationale on which TKI alone may still be a viable first-line treatment option for some subgroups of patients with mRCC.

\section{Monotherapy vs Combination Therapy}

Tumor neo-angiogenesis is a key event in tumor progression, and several drugs with antiangiogenic activity as TKIs or bevacizumab have proven to be effective in firstline treatment [8]. In phase III studies, conducted mainly on good- or intermediate-risk patients, sunitinib showed a stark improvement in progression-free survival (PFS) compared to interferon alfa (IFN- $\alpha)[9,10]$.

In addition, pazopanib has shown non-inferiority to sunitinib in the phase III COMPARZ study [11]. More recently, cabozantinib, a TKI which acts on various receptors such as hepatocyte growth factor receptor, vascular endothelial growth factor receptor (VEGFR), AXL, and FLT3, showing multiple inhibitory effects on angiogenesis, cell proliferation, migration and invasion, and solid tumor growth [12], also showed, as a primary endpoint, prolonged PFS ( 8.6 months, $95 \%$ CI $6.8-14.0$ vs 5.3 months, 95\% CI 3.0-8.2; hazard ratio [HR] 0.48, 95\% CI 0.31-0.74). In this CABOSUN phase II trial, cabozantinib was used as first-line with respect to sunitinib on 157 intermediate and poor-risk patients [13]. Cabozantinib improved PFS - the primary endpoint of the study - (HR 0.51 [95\% CI 0.41-0.66], $p<0.001$ ), overall survival (OS) (21.4 vs 16.5 months, HR 0.66 [95\% CI $0.50-0.85])$, and objective response rate (ORR) (17\% vs $3 \%, p<0.001$ ) (both secondary endpoints), in second- and third-line treatment, compared to everolimus. A favorable clinical outcome was established during the follow-up of the study, irrespective of the type of the first treatment, either VEGFR inhibitor or ICIs [14].

In recent clinical trials, the introduction of TKIs (or antiVEGF agent) in combination with new ICIs that interfere with programmed cell death protein 1 (PD-1) [15] showed remarkable results overall.

The combination of bevacizumab with atezolizumab, a monoclonal antibody against the protein programmed cell death-ligand 1 (PD-L1), has been compared with sunitinib in the IMmotion151 study as a first-line treatment in mRCC PD-L1 positive patients. Primary endpoints were PFS in patients expressing PD-L1 and OS in the intention-to-treat population. In the study, the association bevacizumab + atezolizumab was found to be superior over sunitinib in terms of PFS (11.2 months vs 7.7 months, HR $0.74, p=0.02$ ) in PD-L1 positive patients, while no significant improvement in OS was reported [3•].

Moreover, the randomized, open-label, phase III trial JAVELIN Renal 101 demonstrated an advantage with the association of avelumab + axitinib vs sunitinib in previously untreated patients - PD-L1 positive, as shown by the primary endpoint result (PFS in PD-L1 patients 13.8 vs 7.2 months; HR $0.61 ; p<0.001$ ) and also by ORR in PD-L1 positive (a secondary endpoint, $55.2 \%$ vs $25.5 \%$ ), regardless of patient risk category [4•]. A statistically significant advantage in PFS and ORR has also been reported in the general population. The results for the experimental combination in terms of OS in the PD-L1 population, another primary endpoint, are immature at the moment to be properly discussed [16].

Recently, the efficacy of the combination pembrolizumab-axitinib vs sunitinib was investigated in an openlabel, phase III trial involving 861 patients with previously untreated advanced RCC. Primary endpoints were OS and PFS in the intention-to-treat population. The results for the combination showed a $47 \%$ reduction in death risk and $31 \%$ in disease progression compared to the monotherapy, respectively [17]. Nevertheless, the combination therapy did not show a significant benefit in the subgroup of favorable-risk patients (HR for OS: 0.64; 95\% CI, 0.24-1.68) [17].

The CLEAR study, a multicenter open-label, randomized, phase III trial showed a significantly longer PFS (primary endpoint of the study, median 23.9 vs. 9.2 months; HR for disease progression or death, $0.39 ; 95 \% \mathrm{CI}, 0.32$ to 0.49 ; $p<0.001$ ) for the combination lenvatinib-pembrolizumab vs sunitinib monotherapy; HR for death was 0.66 (95\% CI, 0.49 
to $0.88 ; p=0.005)$. In the same study, significantly better PFS was found for the combination lenvatinib-everolimus (without significant OS difference) as first-line treatment of patients with advanced RCC [7•]. The results of this trial are outstanding and definitely introduce a new first-line option for advanced RCC patients, with possible hints on the lenvatinib's potential immunomodulatory activity [7•]. In this case, the favorable-risk subgroup reached a significant PFS benefit (primary endpoint) but still did not reach significant benefit in terms of OS (secondary endpoint) (HR 1.15; 95\% CI, 0.55-2.40).

The combination nivolumab-cabozantinib is also being tested in a phase III study, the CheckMate 9ER. The results of the study at median follow-up of 18.1 months showed superiority of the combination in terms of PFS (primary endpoint of the study, $16.6 \mathrm{v} 8.3$ months, HR $0.51 ; 95 \%$ CI $0.41-0.64, p<0.001)$, OS at 12 months was $85.7 \%$ vs $75.6 \%$ (secondary endpoint; HR 0.60 [98.89\% CI 0.40-0.89]; $p<0.001$ ), and ORR (secondary endpoint; $55.7 \%$ [50.1-61.2] vs $27.1 \%$ [22.4-32.3]; $p<0.001$ ) compared to sunitinib, in first-line treatment of all the subgroups analyzed. In addition, $8.0 \%$ of the individuals in combination therapy achieved a complete response, compared to $4.6 \%$ in the sunitinib group. These results were also supported by a manageable safety profile [18].

A recent phase III study (CONTACT 03) comparing the combination of cabozantinib and atezolizumab vs cabozantinib monotherapy has already begun as a second-line treatment for patients treated with ICI [19]. This study will test the efficacy and safety (primary endpoints: PFS and OS; secondary endpoints: ORR and safety among others) of the combination vs monotherapy and investigate the efficacy of sequential treatments, in this case, a combination of a TKI and a PD-L1 inhibitor or a TKI alone after an ICI.

Another large phase III study (CHECKMATE 214), conducted on 1096 untreated mRCC patients, demonstrated an improvement in OS and ORR (both primary endpoints) with the combination of two ICIs, nivolumab, targeting PD-L1, and ipilimumab, a monoclonal antibody which inhibits cytotoxic T-lymphocyte-associated antigen 4 (CTLA-4) [20], compared to sunitinib, in intermediate- and poor-risk patients [ $[\bullet \bullet$ ]; these data were confirmed by the follow-up. Of note, the primary endpoint population was the intermediate-poor-risk patient group, the intention-to-treat population including all-risk patients. Following a median of $>32$ months in favorable-risk patients, the PFS and OS were found to be similar between the two arms of treatment in this subgroup, with a greater proportion of patients achieving objective response with sunitinib (50\% vs 39\%, $p=0.14$ ) [21]. Interestingly, the benefit of the combination treatment vs sunitinib was even more evident in intermediate/poor-risk patients with sarcomatoid carcinoma, likely due to the worse prognosis of sarcomatoid cases treated with TKI monotherapy as the comparator. In this sarcomatoid carcinoma subgroup, $56.7 \%$ vs $19.2 \%(p<0.001)$ of the patients reached an ORR and $18.3 \%$ vs $0 \%$ patients showed a complete response with the combination therapy compared to the monotherapy, respectively. These data are sustained by a safe profile, and prospective studies are underway to better the efficacy assessment of nivolumab/ipilimumab in this subgroup of patients [22].

Recently, the phase III TiNivo-2 trial has been announced to test both the activity and the tolerability of the combination tivozanib-nivolumab vs tivozanib monotherapy [23]. Tivozanib is a TKI with a potent action specifically on VEGF-1, 2, 3 [24]. Tivozanib was shown to be effective as a third- or fourth-line treatment compared to sorafenib in phase III TIVO-3 trial [25]. The phase I/II trial showed that the combination tivozanib-nivolumab is comparable to other TKI/PD-1 combinations in terms of frequency and severity of adverse events $[4 \bullet, 17,26]$.

Furthermore, the combination belzutifan (a hypoxiainducible factor $2 \alpha$ inhibitor)-cabozantinib is being evaluated in a phase II trial in advanced RCC patients either naïve or who have already received 1 or 2 previous treatments. Preliminary results for the arm of patients who were already treated indicate good tolerability, with no grade 4 TRAEs registered after 24 months (median time from enrollment to data cutoff 11.3 months, 5.6-24.0 months) [27•, 28].

The trials investigating the combination therapies show promising and exciting results. However, most of the patients enrolled in clinical studies are characterized by overall good performance status and the absence of relevant comorbidities. This condition is most likely unmet in the daily routine, where a quite heterogeneous population is expected to be treated [29]. It has been estimated that oncology accounts for $<5 \%$ of patients enrolled in clinical trials [30], and consequently, the study population does not entirely represent those observed during daily clinical practice.

Therefore, it is necessary to consider specific patient characteristics in order to define the best treatment, as a combination therapy may not always be the optimal choice, and other alternatives, such as the therapy with the TKI alone, may still be considered.

\section{Combination Therapy Versus TKI Alone in Selected Patient Subgroups}

Combination therapy has been shown to be superior to monotherapy in clinical trials $[3 \bullet, 5 \bullet \bullet, 17]$. Nevertheless, there are subgroups of patients for whom the association of agents could not be the optimal choice, resulting not applicable due to the patient characteristics. This is the case for patients who suffer from autoimmune diseases. Additionally, there is a lack of clear evidence of the beneficial 
effects of combination therapy in the subgroup of favorable-risk patients, as shown by the subgroup analyses from pivotal clinical trials. Regarding the group of favorable-risk patients, the Checkmate 9ER trial reports the efficacy of the combination nivolumab plus cabozantinib in all the categories of patients (favorable, intermediate, and poor-risk), although the published data report that the follow-up is still ongoing and the study is not mature to assess the survival in the favorable-risk group [18].

In the KEYNOTE 426 study, the pembrolizumab-axitinib combination showed superiority over sunitinib in terms of OS, PFS, and ORR [17]. However, the follow-up of the study data presented at the ASCO Annual Meeting 2020 showed a lack of maintenance in good-risk patients in terms of PFS and OS, with no significant differences between both combination and monotherapy after 23 months [31].

In addition, the CHECKMATE 214 study with nivolumab/ipilimumab, which reported beneficial effects for most of the patients, failed to show any improvement in those with a favorable prognosis, showing a higher OS in the sunitinib group [5••]. Moreover, first-line cabozantinib monotherapy was effective specifically in poor-intermediate-risk patients, as evident in the phase II CABOSUN trial $[13,32]$.

A recent report compares first-line combination treatments (immune-modulation + anti-VEGF vs ipilimumab + nivolumab) within the intermediate/poor-risk dataset patients of the International mRCC Database Consortium (IMDC), concluding no detectable difference in the OS among the therapies for these subsets of patients [33].

A specific subset of mRCC patients is represented by those with pancreatic or lung metastases only, often characterized by an overall good outcome. Indeed, RCC patients with pancreatic metastases showed a favorable prognosis compared to other sites of metastases [34, 35]. Interestingly, it has been observed that RCCs which metastasize to the pancreas are characterized by a higher sensitivity to antiangiogenic agents and resistance to ICIs [36•]. In a retrospective study involving 262 patients, the probability of survival after pancreatic metastases at 1 , 3 , and 5 years resulted in being $100 \%, 87.7 \%$, and $78.9 \%$, respectively. Compared to patients with non-pancreatic metastasis (median OS 2.7 years, $p<0.0001$ ), the survival rate found in the study was significantly longer [37]. The lung is one of the most common metastatic sites in RCC [38], but their presence is anyway associated with a better outcome than metastases in other sites [39]. Interestingly, an analysis performed on pulmonary metastases characterized by spontaneous regression revealed $76 \%$ of RCC origin [40]. In addition, an observational study comparing OS in patients with pancreatic and lung metastases treated with TKIs alone or treated with surgical resection showed no significant differences between the two groups (OS: 86 vs 103 months respectively, $p=0.201$ ) [41]. Given the same favorable outcome for single therapy as well as surgery for pulmonary metastases, combination therapy may be uncertain for patients with pancreatic or pulmonary disease.

There are other categories of patients for whom using a TKI alone might be advisable rather than a combination therapy [42-44]. A typical example is represented by patients with renal function impairment, usually not included in large prospective trials. The largest case history, including 39 RCC patients with renal function impairment treated with TKI (sunitinib or sorafenib), has been reported by the Cleveland group (USA) [45]. Although dose reduction was necessary for half of the patients due to increased creatinine levels, treatment efficacy remained substantially similar, both in terms of partial response and stable disease, for patients with preserved renal function [45].

It must be mentioned that immunotherapy is not suggested in the case of any autoimmune pathology and its administration must be carefully evaluated for patients in need of chronic immunosuppressant treatment. Therefore, ICIs also must be avoided in patients with autoimmune nephropathy [46]. Furthermore, the use of these agents should be carefully considered in those with a previous organ transplantation history in order to avoid organ rejection due to a strong immune response. Presently, few preclinical data are available in this regard combined with other case reports [47, 48•], with no clear indications from randomized controlled trials.

Elderly patients are also another category garnering a particular interest as half of the newly diagnosed renal cell carcinoma are found in patients $>65$ years of age, predominantly $25 \%$ cases between 65 and 74 , and another $25 \%$ cases in $>75$ years old $[49,50]$. There is a lack of significant data concerning the outcome of combination therapies in this population. Elderly patients in clinical trials are usually poorly represented due to several reasons: (1) a supposed greater risk of adverse events and therefore reduced tolerability of treatments and (2) the presence of comorbidities worsening performance status. Recent data report the comparison of ICI treatments (monotherapy or combination) in older patients with respect to young adults. No association has been found at multivariate analysis between older age and worst OS. However, older population displayed a shorter median OS compared to younger individuals (25.1 vs. 30.8 months) and lower ORR ( $24 \%$ vs. $31 \%, p=0.01)$ likely due to a difference response in first-line treatment ( $31 \%$ vs. $44 \%, p=0.02$ for older and younger, respectively) [51•]. It is not possible to exclude a patient for a combination treatment based only on age [51•]. Nevertheless, elderly patients often present multiple comorbidities or difficulties accessing a treatment center for intravenous therapy on a periodic basis. Therefore, TKI alone could be considered a valid option for elderly patients. 
Indeed, interesting results from the trials have shown the potential use of a single TKI as a therapeutic agent in this population.

In a recent study, around $32-40 \%$ of the patients ( $>65$ years old) enrolled with sunitinib $[9,52,53]$ demonstrated comparable efficacy and adverse events to that of younger patients. A prospective observational study, PRINCIPAL, carried out in a real-world setting including $60 \%$ patients $>65$ years (median age 66 years, range 22-90), confirmed the efficacy of pazopanib in terms of OS, PFS, and ORR [54].

Looking more closely at the age distribution of patients, it can be emphasized that a substantial improvement was observed in second-line PFS obtained with axitinib relative to sorafenib (6.7 vs. 4.7 months, HR $0.665 ; 95 \%$ CI $0.544-0.812$; one-sided $p<0.0001)$ and a low percentage of discontinuation of therapy ( $4 \%$ vs $8 \%$, respectively), in a population where $34 \%$ of the patients were 65 years or older [55]. Similarly, the METEOR study, which included $40 \%$ of patients $>65$ years in second-line settings, showed amelioration in cabozantinib OS, PFS, and ORR compared to everolimus [56].

In light of the presented findings, it is questionable whether it is appropriate to expose specific subgroups (favorable prognosis patients, fragile patients, or subjects with multiple comorbidities) to combination therapy. In terms of saving of resources and toxicity, often preserving a good quality of life, the use of TKI monotherapy is still likely to be suggested for these subgroups of patients to achieve an overall good outcome.

On the other hand, some patients may present contraindications for TKI use. This happens in the case of predisposition to pathologies that are related to TKI-induced adverse events. In particular, hypertension is one of the most common adverse events associated with the use of TKIs, with an incidence of occurrence reported between $17 \%$ and $49.6 \%$ of patients [57]. Therefore, for those subjects already suffering from severe cardiovascular diseases, hypertension induced by TKIs could predispose to a higher risk of worsening of the condition and developing of cardiovascular events [57-59]. The molecular pathways involved in TKI-induced hypertension still need to be elucidated, and a genetic predisposition may be involved [57]. Nevertheless, the development of hypertension seems to be correlated with better TKI efficacy [60]. Specific attention should also be paid to patients at risk of hemorrhages as the TKI in association with anticoagulant therapy has been reported to increase the bleeding risk [61]. Similarly, fragile patients suffering from hepatopathies and elevated transaminase levels [62] or gastrointestinal problems $[63,64]$ may be exposed to a higher risk of exacerbations when treated with a TKI. In such conditions, therapy with an ICI as a first-line followed by a combination of two ICIs as a second-line may be a possible option, as recently explored with a multicentric European study (TITAN-RCC). This trial is designed to assess the outcome of treatment with nivolumab alone, followed by the addition of ipilimumab as a booster to improve the efficacy of the treatment and to reduce the rate of adverse events [65]. The association of PD-1 and CTLA-4 inhibitors may be better tolerated than TKI in some patients, but there are currently no factors predicting for immune-related toxicity to guide the clinical decision [66•].

\section{Immunomodulation by TKIs: Influence on Sequence and Combination Therapy}

RCC is considered an immunogenic tumor, and a high number of immune cells are detectable within the tumor tissue, such as tumor-infiltrating lymphocytes (Fig. 1) [67, 68•]. Cancer development is delayed by mounting an effective immune response to the tumor [69]. Antiangiogenic agents have been shown to delay tumor progression not only by impairing angiogenesis in the tumor microenvironment but also by dampening the immune response of immunosuppressive cytokines and cells, like T reg cells [70,71]. Since most TKIs have antiangiogenic capabilities, they can also boost the immune response. In this scenario, a TKI monotherapy can provide the required immune priming against the tumor, even without using ICI. Evidence of immune priming due to the modulation of angiogenetic molecules, in particular VEGF, has been provided by different studies, both directly (inhibition of the maturation of dendritic cells and proliferation of effector T cells, with up-regulation of PD-L1
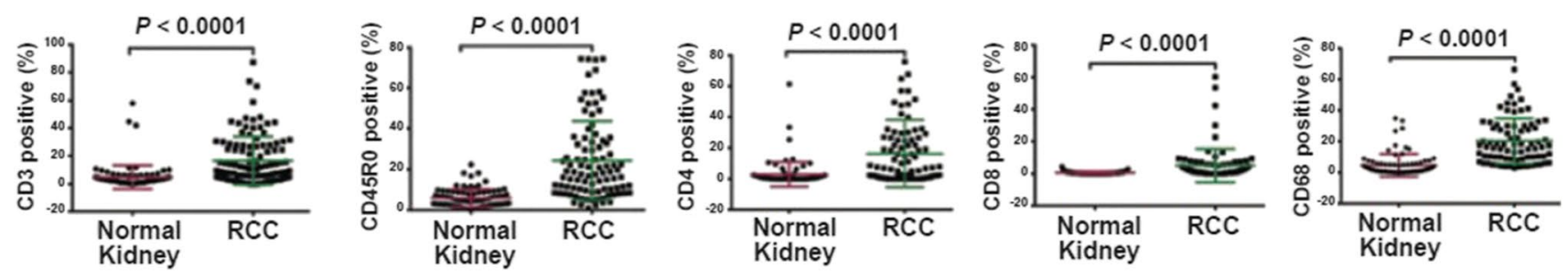

Fig. 1 Immune cells in RCC, compared with normal kidney tissue. RCC, renal cell carcinoma. Modified from [67] 
expression, reduction of $\mathrm{T}$ reg cells) and indirectly (modulation of adhesion molecules and chemokine expression which in turn decreases the recruitment of immune cells within the tumor) $[72,73]$. Bevacizumab and the combination bevacizumab-atezolizumab have been shown to reduce the level of inflammatory cytokines (IFN $\gamma$, IL-4, and IL-17), improve in vitro and in vivo cytotoxic T-lymphocyte response, and promote dendritic cell activation [74] and T-lymphocyte migration [75].

Furthermore, axitinib increases effective immune cell infiltration into the tumor tissue [76], cabozantinib actively downregulates suppressive myeloid cells and stimulates T-effector cells [77], and $\mathrm{T}$ regs appear to be decreased by sunitinib as well [78].

Interestingly, pazopanib also appears to exhibit immunomodulatory actions. Verzoni et al. conducted a study on 16 patients treated with pazopanib as first-line for mRCC, analyzing blood immune profiling after 6 months of therapy. The drug showed a marked reduction in immune-suppressive myeloid cells, like $\mathrm{T}$ reg and myeloid-derived suppressor cells (Fig. 2).

These results were also supported by a study conducted in vitro on DCs from healthy donors [79••]. DCs from healthy subjects showed an increase of differentiation rate when exposed to pazopanib and exhibited greater $\mathrm{T}$ cells stimulation.
Data were confirmed by the clinical phase of the study, in which treatment with pazopanib significantly increased DC activation and a greater down-regulation of DC-regulated PD-L1 and IL-10 production [79••].

The immunomodulation activity can also be achieved through the reprogramming of the metabolism of the tumor cell. Indeed, RCC is characterized by a modification of several biochemical, cellular pathways. A switch toward increased glucose utilization (via glycolysis, glycogenolysis, pentose phosphate pathway) and an impairment of both oxidative phosphorylation and fatty acid metabolism, both mitochondrial pathways, is a signature of RCC cancer cells [80-83]. The metabolic signature of renal cancer cells is also characterized by increased use of amino acids such as glutamine and glutamate $[80,83]$. As a consequence of this metabolic reprogramming, the tumor microenvironment displays a peculiar composition with several metabolic intermediates that can influence the response to treatment. The role played by metabolism in cancer therapies emerged in a study where, upon nivolumb treatment, a specific response - increased serum kynurenine/tryptophan ratio - was associated with a worse outcome [84]. Moreover, the metabolic-modified tumor microenvironment may interfere with the behavior of the surrounding cells including immune cells. In fact, the administration of an adenosine receptor antagonist, a metabolic modulator, was associated with $\mathrm{CD} 8+\mathrm{T}$ cells infiltration in tumor biopsies,
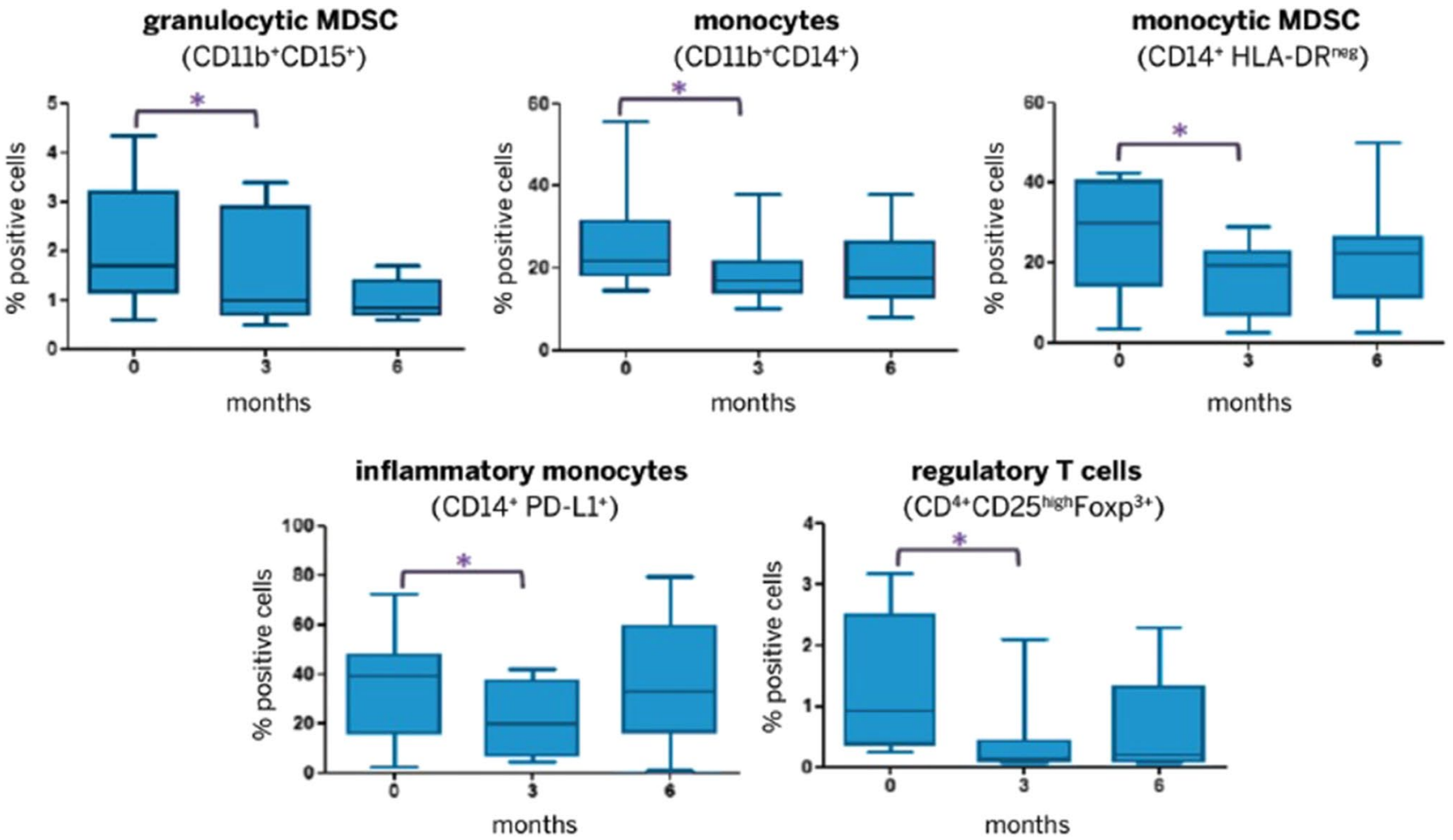

Fig. 2 Reduction of immunosuppressive cells (MDSC and T reg) in peripheral blood during treatment with pazopanib for RCC. MDSC, myeloid-derived suppressor cells. Modified from [77] 
underlying an interplay between metabolic pathways modulation and immune cell infiltration [68•]. The activation of specific metabolic pathways and the respective accumulation or depletion of intermediates can regulate angiogenesis and inflammatory features, thus influencing tumor fate [85]. The targeting of metabolic pathways is a field yet to be explored and may represent a new frontier in combination therapies with TKI, mTOR inhibitors, and ICI agents for the treatment of RCC [86].

In a sequential therapy rather than a combination, TKI's ability to stimulate immune response could be an advantage. Some patients may benefit from immune priming first, initial tumor response, and specific immunotherapy subsequently. The scarcity of reports has made it difficult to assess which treatment option would be the best for triggering an immune response, whether TKI and ICI are used sequentially or TKI and immunotherapy are used simultaneously. To date, prospective data regarding the efficacy of different sequencing therapies are not available, as most of the studies are still ongoing and the data are therefore immature to draw any conclusion. One example is the Breakpoint Study, assessing the role of cabozantinib in patients pretreated with one ICI in monotherapy or in combination [87]. The evidence regarding the efficacy of targeted agents in patients pretreated with ICIs comes from retrospective analyses $[88 \bullet \bullet, 89 \bullet \bullet]$.

ICIs can then synergize with antiangiogenic/TKIs [73] since there is a clear rationale for their use in the treatment of multiple tumors, including RCC [90].

Indeed, the expression of immune checkpoint receptors, particularly CTLA-4 and PD-1/PD-L1, represent pathways by which T-cell immunity is blocked [91]. Hence, they represent potential therapeutic targets, and their inhibition has led to a significant clinical benefit in the treatment of several types of tumors [73, 78, 92-94]. For mRCC patients, the combination of various immunotherapy agents seems to be a reliable alternative.

However, a variable proportion of patients (about $20-40 \%$ ) are primary refractory to ICIs [95], and immunotherapy is useless for this subset of patients. In the absence of predictive factors that can identify these primary refractory patients, giving an extra drug could lead to a risk of additional toxicity without a clinically realistic benefit to the patient. In this case, the use of TKI monotherapy which could stimulate the immune response is a good alternative.

\section{Gut Microbiota Modulation in RCC}

Gut microbiota has recently been included as a modulating immunotherapy factor that can influence the response to cancer therapy $[96,97 \bullet]$. The microbiota plays a fundamental role in immune functions $[98,99]$. Its alterations can cause anomalies in local and systemic immune responses, affecting cytokine secretion and T lymphocytes activation [97•, 100]. Specifically, alterations of the microbiota can influence the response and toxicity of antineoplastic therapies, as demonstrated by a large number of both preclinical $[101,102]$ and clinical [96, 103-106] data.

The impact of intestinal microbiota on the outcomes of treatment with ICIs is fascinating. A proportion of patients treated with ICIs have no, or not durable, responses. Emerging evidence suggests that alterations in the intestinal microbiota (often caused by previous therapies) can be included among the factors that affect immunotherapy resistance [104].

Gopalakrishnan et al. demonstrated that, relative to nonresponders, patients responding to anti-PD-1 therapy for metastatic melanoma have a more differentiated microbiota [96].

Any alterations in the intestinal microbiome caused by previous therapies, such as TKIs, should be addressed to allow the restoration of physiological conditions. Ianiro et al. have reported the treatment of diarrhea caused by TKIs in RCC patients following fecal microbiota transplantation. It can be hypothesized that the results of subsequent therapies, particularly with ICIs, may be influenced by this manipulation of microbiota [107•]. Therefore, it is an important factor in defining personalized anticancer therapy, considering the wide range of individual variations, including differences in intestinal microbiota.

\section{Conclusions}

The treatment and management of metastatic RCC patients have radically changed over the past 20 years [108••]. A great number of trials have demonstrated the efficacy of numerous molecules and combinations, prolonging the PFS and OS of RCC patients. An increased number of combination therapies, associating either an immune agent and TKI or two immune agents, represent new opportunities for RCC treatment and are beneficial for most patients. However, it is not excluded that some selected categories of patients may still benefit from monotherapy with TKI and a smart treatment sequence instead of a combination strategy. These patients may be a minority but still represented within the population: the ones with favorable risk and very long survival due to indolent disease, the ones with autoimmune pathologies, those normally excluded by clinical studies (patients with ECOG score $>1$, patients with relevant cardiac or pulmonary comorbidities, renal impairment, elderly patients or patients with only pulmonary or pancreatic metastases), who are the real-world patients.

As the immunological response is essential in RCC, an effective immune stimulation could improve the patient 
outcome. Some TKIs have been shown to have immunomodulatory activity, and their use can prove beneficial in boosting the patient immune response against the tumor, even more, if preceding ICIs in the treatment sequence.

Currently, there is insufficient evidence to adopt the best strategy for promoting immune response, as some patients may benefit from a sequential therapy with a TKI followed by immunotherapy, while others may require a combination treatment TKI-immunotherapy upfront.

It is imperative to define the most appropriate treatment for each patient, and in some selected conditions, the use of TKIs can still represent a therapeutic option.

The available data, albeit limited, could provide a rationale for further studies aimed (e.g., by searching for specific markers) at identifying patients suitable for sequential TKI-immunotherapy.

Acknowledgements Medical writing support was provided by Barbara Bartolini on behalf of Health Publishing \& Services Srl, which was funded by Novartis Farma SpA. The funder was not involved in the conception, drafting, and writing of this manuscript or in the decision to submit it for publication.

Data Availability Not applicable.

Code Availability Not applicable.

\section{Declarations}

Competing Interests E.R.: consultant for MSD. M.B. received research funding by Roche, Novartis, Pfizer, and Seqirus, fees for advisory role and speakers' bureau by AstraZeneca, IPSEN, Novartis, Pierre Fabre, Pfizer, and BMS, and honoraria for copyright transfer by Sciclone Pharmaceuticals. E.V. received speakers' bureau/advisory board fees from Pfizer, MSD, Novartis, Ipsen, and BMS. P.A.Z. reports outside the submitted work personal fees for advisory role, speaker engagements, and travel and accommodation expenses from Merck Sharp \& Dohme, Astellas, Janssen, Sanofi, Ipsen, Pfizer, Novartis, Bristol Meyer Squibb, Amgen, AstraZeneca, Roche, and Bayer. The other authors declare no conflict of interest.

Open Access This article is licensed under a Creative Commons Attribution 4.0 International License, which permits use, sharing, adaptation, distribution and reproduction in any medium or format, as long as you give appropriate credit to the original author(s) and the source, provide a link to the Creative Commons licence, and indicate if changes were made. The images or other third party material in this article are included in the article's Creative Commons licence, unless indicated otherwise in a credit line to the material. If material is not included in the article's Creative Commons licence and your intended use is not permitted by statutory regulation or exceeds the permitted use, you will need to obtain permission directly from the copyright holder. To view a copy of this licence, visit http://creativecommons.org/licen ses/by/4.0/.

\section{References}

Papers of particular interest, published recently, have been highlighted as:

- Of importance

$\bullet \bullet$ Of major importance

1.• Hahn AW, Klaassen Z, Agarwal N, et al. First-line treatment of metastatic renal cell carcinoma: a systematic review and network meta-analysis. Eur Urol Oncol. 2019;2:708-15. https:// doi.org/10.1016/j.euo.2019.09.002. (A network-meta analysis designed to compare the efficacy and safety of first-line therapies in $\mathrm{mRCC}$ patients in different clinical risk subgroups.)

2. Barata PC, Rini BI. Treatment of renal cell carcinoma: current status and future directions. CA Cancer J Clin. 2017;67:507-24. https://doi.org/10.3322/caac.21411.

3. Rini BI, Powles T, Atkins MB, et al. Atezolizumab plus bevacizumab versus sunitinib in patients with previously untreated metastatic renal cell carcinoma (IMmotion151): a multicentre, open-label, phase 3, randomised controlled trial. Lancet. 2019;393:2404-15. https://doi.org/10.1016/S0140-6736(19) 30723-8. (The clinical trial showing the evidence of longer progression-free survival in RCC for the combination atezolizumab plus bevacizumab vs sunitinib.)

4. Motzer RJ, Penkov K, Haanen J, et al. Avelumab plus axitinib versus sunitinib for advanced renal-cell carcinoma. N Engl J Med. 2019;380:1103-15. https://doi.org/10.1056/NEJMoa1816 047. (The phase III trial showing the superiority of fist-line treatment with avelumab plus axitinib versus sunitinib in progression-free survival for advanced $\mathrm{RCC}$ )

5.•• Motzer RJ, Tannir NM, Mcdermott DF, et al. Nivolumab plus ipilimumab versus sunitinib in advanced renal-cell carcinoma. $\mathrm{N}$ Engl J Med. 2018;378:1277-90. https://doi.org/10.1056/NEJMo a1712126. (This is the first phase III trial reporting the better outcome (in terms of overall survival and objective response rate) of combination therapy as a first-line treatment of advanced RCC intermediate- and poor-risk patients.)

6. Choueiri TK, Powles T, Burotto M, Bourlon MT, Zurawski B, Oyervides Juárez VM, Hsieh JJ, Basso U, Shah AY, Suarez C, Hamzaj A, Barrios CH, Richardet M, Pook D, Tomita Y, Escudier B, Zhang J, Simsek B, Apolo AB, Motzer RJ. Nivolumab + cabozantinib vs sunitinib in first-line treatment for advanced renal cell carcinoma: first results from the randomized phase III CheckMate 9ER trial. Annals of Oncology. 2020;31(4):S1142-215.

7. Motzer R, Alekseev B, Rha SY, et al. Lenvatinib plus Pembrolizumab or everolimus for advanced renal cell carcinoma. N Engl J Med. 2021. https://doi.org/10.1056/NEJMoa2035716. (This study shows the evidence of the efficacy of lenvatinib plus pembrolizumab vs sunitinib in progression-free survival and overall survival of RCC patients.)

8. Haaland B, Chopra A, Acharyya S, et al. Comparative effectiveness of approved first-line anti-angiogenic and molecularly targeted therapeutic agents in the treatment of good and intermediate risk metastatic clear cell renal cell carcinoma. BMC Cancer. 2014;14:592. https://doi.org/10.1186/1471-2407-14-592.

9. Motzer RJ, Hutson TE, Tomczak P, et al. Sunitinib versus interferon alfa in metastatic renal-cell carcinoma. N Engl J Med. 2007;356:115-24. https://doi.org/10.1056/NEJMoa065044.

10. Motzer RJ, Hutson TE, Tomczak P, et al. Overall survival and updated results for sunitinib compared with interferon alfa in patients with metastatic renal cell carcinoma. J Clin Oncol. 2009;27:3584-90. https://doi.org/10.1200/JCO.2008.20.1293. 
11. Motzer RJ, Hutson TE, Cella D, et al. Pazopanib versus sunitinib in metastatic renal-cell carcinoma. N Engl J Med. 2013;369:722-31. https://doi.org/10.1056/NEJMoa1303989.

12. Yakes FM, Chen J, Tan J, et al. Cabozantinib (XL184), a novel MET and VEGFR2 inhibitor, simultaneously suppresses metastasis, angiogenesis, and tumor growth. Mol Cancer Ther. 2011;10:2298-308. https://doi.org/10.1158/1535-7163. MCT-11-0264

13. Choueiri TK, Hessel C, Halabi S, et al. Cabozantinib versus sunitinib as initial therapy for metastatic renal cell carcinoma of intermediate or poor risk (Alliance A031203 CABOSUN randomised trial): progression-free survival by independent review and overall survival update. Eur J Cancer. 2018;94:115-25. https://doi.org/10.1016/j.ejca.2018.02.012.

14. Powles T, Motzer RJ, Escudier B, et al. Outcomes based on prior therapy in the phase 3 METEOR trial of cabozantinib versus everolimus in advanced renal cell carcinoma. Br J Cancer. 2018;119:663-9. https://doi.org/10.1038/s41416-018-0164-0.

15. Hahn AW, Gill DM, Pal SK, et al. The future of immune checkpoint cancer therapy after PD-1 and CTLA-4. Immunotherapy. 2017;9:681-92. https://doi.org/10.2217/imt-2017-0024.

16. Choueiri TK, Motzer RJ, Rini BI, et al. Updated efficacy results from the JAVELIN Renal 101 trial: first-line avelumab plus axitinib versus sunitinib in patients with advanced renal cell carcinoma. Ann Oncol. 2020;31:1030-9. https://doi.org/10.1016/j. annonc.2020.04.010.

17. Rini BI, Plimack ER, Stus V, et al. Pembrolizumab plus axitinib versus sunitinib for advanced renal-cell carcinoma. $\mathrm{N}$ Engl $\mathbf{J}$ Med. 2019;380:1116-27. https://doi.org/10.1056/NEJMoa1816 714.

18. Choueiri TK, Powles T, Burotto M, et al. Nivolumab plus cabozantinib versus sunitinib for advanced renal-cell carcinoma. $\mathrm{N}$ Engl J Med. 2021;384:829-41. https://doi.org/10.1056/NEJMo a2026982.

19. https://clinicaltrials.gov/ct2/show/NCT04338269?term=NCT04 $338269 \&$ draw $=2 \&$ rank $=1$. [Accessed on 3 April 2021].

20. Funt SA, Page DB, Wolchok JD, et al. CTLA-4 antibodies: new directions, new combinations. Oncology (Williston Park). 2014;28(Suppl 3):6-14.

21. Motzer RJ, Rini BI, Mcdermott DF, et al. Nivolumab plus ipilimumab versus sunitinib in first-line treatment for advanced renal cell carcinoma: extended follow-up of efficacy and safety results from a randomised, controlled, phase 3 trial. Lancet Oncol. 2019;20:1370-85. https://doi.org/10.1016/S1470-2045(19) 30413-9.

22. Mcdermott Df CT, Motzer Rj, Aren or, George S, Powles T, Donskov F, Harrison Mr, Rodriguez Cid Jrr, Ishii Y, Mchenry Mb, Mekan Sf, Rini Bi, https://doi.org/10.1200/Jco.2019.37. 15_Suppl.4513 Journal of Clinical Oncology 37, No. 15_Suppl (May 20, 2019) 4513-4513.Tannir Nm, CheckMate 214 posthoc analyses of nivolumab plus ipilimumab or sunitinib in IMDC intermediate/poor-risk patients with previously untreated advanced renal cell carcinoma with sarcomatoid features. 2019

23. https://www.urologytimes.com/view/nivolumab-plus-tivozanibexplored-in-phase-3-kidney-cancer-trial. [Accessed on 3 April 2021].

24. Saes L, Eskens F. Tivozanib: a new treatment option for renal cell carcinoma. Drugs Today (Barc). 2017;53:609-18. https:// doi.org/10.1358/dot.2017.53.11.2724804.

25. Rini BI, Pal SK, Escudier BJ, et al. Tivozanib versus sorafenib in patients with advanced renal cell carcinoma (TIVO-3): a phase 3, multicentre, randomised, controlled, open-label study. Lancet Oncol. 2020;21:95-104. https://doi.org/10.1016/S14702045(19)30735-1.

26. Albiges L, Barthelemy P, Gross-Goupil M, et al. TiNivo: safety and efficacy of tivozanib-nivolumab combination therapy in patients with metastatic renal cell carcinoma. Ann Oncol. 2021;32:97-102. https://doi.org/10.1016/j.annonc.2020.09.021.

27. Choueiri TK, Bauer TM, Mcdermott DF, et al. Phase 2 study of the oral hypoxia-inducible factor $2 \alpha$ (HIF-2 $\alpha$ ) inhibitor MK-6482 in combination with cabozantinib in patients with advanced clear cell renal cell carcinoma (ccRCC). J Clin Oncol. 2021;39:272. (A recent phase II study investigating a new combination therapy in RCC: a transcription factor inhibitor plus a TKI.)

28. https://clinicaltrials.gov/ct2/show/NCT03634540. [Accessed on 3 April 2021].

29. Goebell PJ, Staehler M, Muller L, et al. Changes in treatment reality and survival of patients with advanced clear cell renal cell carcinoma - analyses from the German Clinical RCC-Registry. Clin Genitourin Cancer. 2018;16:e1101-15. https://doi.org/10. 1016/j.clgc.2018.06.006.

30. Murthy VH, Krumholz HM, Gross CP. Participation in cancer clinical trials: race-, sex-, and age-based disparities. JAMA. 2004;291:2720-6. https://doi.org/10.1001/jama.291.22.2720.

31. Jacob A, Shook J, Hutson TE. Tivozanib, a highly potent and selective inhibitor of VEGF receptor tyrosine kinases, for the treatment of metastatic renal cell carcinoma. Future Oncol. 2020;16:2147-64. https://doi.org/10.2217/fon-2020-0443.

32. Choueiri TK, Halabi S, Sanford BL, et al. Cabozantinib versus sunitinib as initial targeted therapy for patients with metastatic renal cell carcinoma of poor or intermediate risk: the alliance A031203 CABOSUN Trial. J Clin Oncol. 2017;35:591-7. https://doi.org/10.1200/JCO.2016.70.7398.

33. Gan CL, Dudani S, Wells JC, et al. Outcomes of first-line (1L) immuno-oncology (IO) combination therapies in metastatic renal cell carcinoma (mRCC): results from the International mRCC Database Consortium (IMDC). J Clin Oncol. 2021;39:276-276. https://doi.org/10.1200/JCO.2021.39.6_ suppl.276.

34. Kalra S, Atkinson BJ, Matrana MR, et al. Prognosis of patients with metastatic renal cell carcinoma and pancreatic metastases. BJU Int. 2016;117:761-5. https://doi.org/10.1111/bju. 13185.

35. Grassi P, Verzoni E, Mariani L, et al. Prognostic role of pancreatic metastases from renal cell carcinoma: results from an Italian center. Clin Genitourin Cancer. 2013;11:484-8. https://doi.org/ 10.1016/j.clgc.2013.04.022.

36. Singla N, Xie Z, Zhang Z, et al., Pancreatic tropism of metastatic renal cell carcinoma. JCI Insight, 2020;5: https://doi.org/10. 1172/jci.insight.134564. (A study investigating the reason(s) why pancreatic metastasis is associated with improved survival in RCC patients, pointing out that these kinds of tumors are resistant to ICIs and are specifically sensitive to antiangiogenic agents)

37. Yuasa T, Inoshita N, Saiura A, et al. Clinical outcome of patients with pancreatic metastases from renal cell cancer. BMC Cancer. 2015;15:46. https://doi.org/10.1186/s12885-015-1050-2.

38. Chen F, Fujinaga T, Shoji T, et al. Pulmonary resection for metastasis from renal cell carcinoma. Interact Cardiovasc Thorac Surg. 2008;7:825-8. https://doi.org/10.1510/icvts.2008.181065.

39. Flanigan RC, Salmon SE, Blumenstein BA, et al. Nephrectomy followed by interferon alfa- $2 \mathrm{~b}$ compared with interferon alfa-2b alone for metastatic renal-cell cancer. N Engl J Med. 2001;345:1655-9. https://doi.org/10.1056/NEJMoa003013.

40. Kumar T, Patel N, Talwar A. Spontaneous regression of thoracic malignancies. Respir Med. 2010;104:1543-50. https://doi.org/ 10.1016/j.rmed.2010.04.026.

41. Santoni M, Conti A, Partelli S, et al. Surgical resection does not improve survival in patients with renal metastases to the pancreas in the era of tyrosine kinase inhibitors. Ann Surg Oncol. 2015;22:2094-100. https://doi.org/10.1245/s10434-014-4256-7. 
42. Feldman DR, Baum MS, Ginsberg MS, et al. Phase I trial of bevacizumab plus escalated doses of sunitinib in patients with metastatic renal cell carcinoma. J Clin Oncol. 2009;27:1432-9. https://doi.org/10.1200/JCO.2008.19.0108.

43. Hainsworth JD, Spigel DR, Burris HA 3rd, et al. Phase II trial of bevacizumab and everolimus in patients with advanced renal cell carcinoma. J Clin Oncol. 2010;28:2131-6. https://doi.org/ 10.1200/JCO.2009.26.3152.

44. Harzstark AL, Small EJ, Weinberg VK, et al. A phase 1 study of everolimus and sorafenib for metastatic clear cell renal cell carcinoma. Cancer. 2011;117:4194-200. https://doi.org/10.1002/ cncr.25931.

45. Khan G, Golshayan A, Elson P, et al. Sunitinib and sorafenib in metastatic renal cell carcinoma patients with renal insufficiency. Ann Oncol. 2010;21:1618-22. https://doi.org/10.1093/annonc/ mdp603.

46. Wanchoo R, Karam S, Uppal NN, et al. Adverse renal effects of immune checkpoint inhibitors: a narrative review. Am J Nephrol. 2017;45:160-9. https://doi.org/10.1159/000455014.

47. Boils CL, Aljadir DN, Cantafio AW. Use of the PD-1 pathway inhibitor nivolumab in a renal transplant patient with malignancy. Am J Transplant. 2016;16:2496-7. https://doi.org/10. 1111/ajt.13786.

48. Abdel-Wahab N, Safa H, Abudayyeh A, et al. Checkpoint inhibitor therapy for cancer in solid organ transplantation recipients: an institutional experience and a systematic review of the literature. J Immunother Cancer. 2019;7:106. https://doi.org/10.1186/ s40425-019-0585-1. (A retrospective analysis evaluating the safe use of ICIs in cancer patients subjected to solid organ transplantation, concluding that the use of these agents is associated with higher rates of allograft rejection, graft loss, and mortality.)

49. Ramos-Barcelo E, Rioja J, Pes PL, et al. Is age a prognostic factor for treatment outcome in renal cell cancer - a comprehensive review. Crit Rev Oncol Hematol. 2009;72:83-9. https://doi.org/ 10.1016/j.critrevonc.2009.02.005.

50. Qayyum T, Oades G, Horgan P, et al. The epidemiology and risk factors for renal cancer. Curr Urol. 2013;6:169-74. https://doi. org/10.1159/000343534.

51. Araujo DV, Wells JC, Hansen AR, et al. Efficacy of immunecheckpoint inhibitors (ICI) in the treatment of older adults with metastatic renal cell carcinoma (mRCC) - an International mRCC Database Consortium (IMDC) analysis. J Geriatr Oncol. 2021. https://doi.org/10.1016/j.jgo.2021.02.022. (In this study, multivariate analysis performed on the data from the International mRCC Database Consortium showed that older age should not preclude the use of ICIs in RCC patients.)

52. Gore ME, Szczylik C, Porta C, et al. Safety and efficacy of sunitinib for metastatic renal-cell carcinoma: an expanded-access trial. Lancet Oncol. 2009;10:757-63. https://doi.org/10.1016/ S1470-2045(09)70162-7.

53. Sternberg CN, Calabro F, Bracarda S, et al. Safety and efficacy of sunitinib in patients from Italy with metastatic renal cell carcinoma: final results from an expanded-access trial. Oncology. 2015;88:273-80. https://doi.org/10.1159/000369256.

54. Schmidinger M, Bamias A, Procopio G, et al. Prospective observational study of pazopanib in patients with advanced renal cell carcinoma (PRINCIPAL study). Oncologist. 2019;24:491-7. https://doi.org/10.1634/theoncologist.2018-0787.

55. Rini BI, Escudier B, Tomczak P, et al. Comparative effectiveness of axitinib versus sorafenib in advanced renal cell carcinoma (AXIS): a randomised phase 3 trial. Lancet. 2011;378:1931-9. https://doi.org/10.1016/S0140-6736(11)61613-9.

56. Choueiri TK, Escudier B, Powles T, et al. Cabozantinib versus everolimus in advanced renal cell carcinoma (METEOR): final results from a randomised, open-label, phase 3 trial.
Lancet Oncol. 2016;17:917-27. https://doi.org/10.1016/S14702045(16)30107-3.

57. Semeniuk-Wojtas A, Lubas A, Stec R, et al., Influence of tyrosine kinase inhibitors on hypertension and nephrotoxicity in metastatic renal cell cancer patients. Int J Mol Sci, 2016;17:https:// doi.org/10.3390/ijms 17122073

58. Kadowaki $\mathrm{H}$, Ishida J, Akazawa $\mathrm{H}$, et al. Axitinib induces and aggravates hypertension regardless of prior treatment with tyrosine kinase inhibitors. Circ Rep. 2021;3:234-40. https://doi.org/ 10.1253/circrep.CR-21-0008.

59. Goldman A, Bomze D, Dankner R, et al. Cardiovascular toxicities of antiangiogenic tyrosine kinase inhibitors: a retrospective, pharmacovigilance study. Target Oncol. 2021. https://doi.org/10. 1007/s11523-021-00817-2.

60. Gadd M, Pranavan G, Malik L. Association between tyrosine-kinase inhibitor induced hypertension and treatment outcomes in metastatic renal cancer. Cancer Rep (Hoboken). 2020;3:e1275. https://doi.org/10.1002/cnr2.1275.

61. Patel SH, George TL, Wang TF, et al. Increased bleeding risk associated with concurrent vascular endothelial growth factor receptor tyrosine kinase inhibitors and low-molecular-weight heparin. Cancer. 2021;127:938-45. https://doi.org/10.1002/ cncr.33337.

62. Keisner SV, Shah SR. Pazopanib: the newest tyrosine kinase inhibitor for the treatment of advanced or metastatic renal cell carcinoma. Drugs. 2011;71:443-54. https://doi.org/10.2165/ 11588960-000000000-00000.

63. Manz KM, Fenchel K, Eilers A, et al. Efficacy and safety of approved first-line tyrosine kinase inhibitor treatments in metastatic renal cell carcinoma: a network meta-analysis. Adv Ther. 2020;37:730-44. https://doi.org/10.1007/ s12325-019-01167-2.

64. Ravaud A. Treatment-associated adverse event management in the advanced renal cell carcinoma patient treated with targeted therapies. Oncologist. 2011;16(Suppl 2):32-44. https://doi.org/ 10.1634/theoncologist.2011-S2-32.

65. Grimm MO, Schmidinger M, Duran Martinez I, Schinzari G, Esteban E, Schmitz M, Schumacher U, Baretton G, Barthelemy P, Melichar B, Charnley N, Schrijvers D, Albiges L. Tailored immunotherapy approach with nivolumab in advanced renal cell carcinoma (TITAN-RCC). Ann Oncol. 2019;30:V892. https:// doi.org/10.1093/annonc/mdz394.051.

66. Abou Alaiwi S, Xie W, Nassar AH, et al., Safety and efficacy of restarting immune checkpoint inhibitors after clinically significant immune-related adverse events in metastatic renal cell carcinoma. J Immunother Cancer, 2020;8: https://doi.org/10.1136/ jitc-2019-000144. (The use of ICIs is associated with immunerelated adverse events. This multicenter retrospective study aimed to evaluate whether restarting an ICI therapy after experiencing adverse events can be safe and effective in RCC patients.)

67. Liu XD, Hoang A, Zhou L, et al. Resistance to antiangiogenic therapy is associated with an immunosuppressive tumor microenvironment in metastatic renal cell carcinoma. Cancer Immunol Res. 2015;3:1017-29. https://doi.org/10.1158/2326-6066. CIR-14-0244.

68. Vuong L, Kotecha RR, Voss MH, et al. Tumor microenvironment dynamics in clear-cell renal cell carcinoma. Cancer Discov. 2019;9:1349-57. https://doi.org/10.1158/2159-8290.CD19-0499. (This is a detailed review of the role of the tumor microenvironment and therein infiltrated immune cells in response to TKI and ICI therapies in RCC.)

69. Disis ML. Immune regulation of cancer. J Clin Oncol. 2010;28:4531-8. https://doi.org/10.1200/JCO.2009.27.2146.

70. Tartour E, Pere H, Maillere B, et al. Angiogenesis and immunity: a bidirectional link potentially relevant for the monitoring of 
antiangiogenic therapy and the development of novel therapeutic combination with immunotherapy. Cancer Metastasis Rev. 2011;30:83-95. https://doi.org/10.1007/s10555-011-9281-4.

71. Heine A, Held SA, Bringmann A, et al. Immunomodulatory effects of anti-angiogenic drugs. Leukemia. 2011;25:899-905. https://doi.org/10.1038/leu.2011.24.

72. Griffioen AW, Damen CA, Martinotti S, et al. Endothelial intercellular adhesion molecule-1 expression is suppressed in human malignancies: the role of angiogenic factors. Cancer Res. 1996;56:1111-7.

73. Hato T, Zhu AX, Duda DG. Rationally combining anti-VEGF therapy with checkpoint inhibitors in hepatocellular carcinoma. Immunotherapy. 2016;8:299-313. https://doi.org/10.2217/imt. 15.126

74. Martino EC, Misso G, Pastina P, et al. Immune-modulating effects of bevacizumab in metastatic non-small-cell lung cancer patients. Cell Death Discov. 2016;2:16025. https://doi.org/10. 1038/cddiscovery.2016.25.

75. Wallin JJ, Bendell JC, Funke R, et al. Atezolizumab in combination with bevacizumab enhances antigen-specific T-cell migration in metastatic renal cell carcinoma. Nat Commun. 2016;7:12624. https://doi.org/10.1038/ncomms12624.

76. Du Four S, Maenhout SK, De Pierre K, et al. Axitinib increases the infiltration of immune cells and reduces the suppressive capacity of monocytic MDSCs in an intracranial mouse melanoma model. Oncoimmunology. 2015;4:e998107. https:// doi.org/10.1080/2162402X.2014.998107.

77. Verzoni EC, A. Squarcina, P. De Cecco, L. Rinchai, D. Bedognetti, D. Grassi, P. Ratta, R. Procopio, G. Rivoltini, L., Broad immunomodulating effect of first-line Pazopanib in metastatic renal cell carcinoma patients. Annals of Oncology 2017 Oct;28 Suppl 6:vi17 2017;28:https://doi.org/10.1093/ annonc/mdx371.043

78. Finke JH, Rini B, Ireland J, et al. Sunitinib reverses type-1 immune suppression and decreases T-regulatory cells in renal cell carcinoma patients. Clin Cancer Res. 2008;14:6674-82. https://doi.org/10.1158/1078-0432.CCR-07-5212.

79.• Zizzari IG, Napoletano C, Botticelli A, et al. TK inhibitor pazopanib primes DCs by downregulation of the beta-catenin pathway. Cancer Immunol Res. 2018;6:711-22. https://doi.org/ 10.1158/2326-6066.CIR-17-0594. (This paper analyzes the immunomodulatory effect of a TKI, suggesting that this treatment could have broader effects than just the inhibition of a biochemical pathway. The action on dendritic cells priming is described both in vitro and in vivo.)

80. Lucarelli G, Loizzo D, Franzin R, et al. Metabolomic insights into pathophysiological mechanisms and biomarker discovery in clear cell renal cell carcinoma. Expert Rev Mol Diagn. 2019;19:397-407. https://doi.org/10.1080/14737159.2019. 1607729 .

81 Bianchi C, Meregalli C, Bombelli S, et al. The glucose and lipid metabolism reprogramming is grade-dependent in clear cell renal cell carcinoma primary cultures and is targetable to modulate cell viability and proliferation. Oncotarget. 2017;8:11350215. https://doi.org/10.18632/oncotarget.23056.

82. Ragone R, Sallustio F, Piccinonna S, et al., Renal cell carcinoma: a study through NMR-based metabolomics combined with transcriptomics Diseases. 2016;4:https://doi.org/10.3390/diseases40 10007

83. Lucarelli G, Rutigliano M, Sallustio F, et al. Integrated multiomics characterization reveals a distinctive metabolic signature and the role of NDUFA4L2 in promoting angiogenesis, chemoresistance, and mitochondrial dysfunction in clear cell renal cell carcinoma. Aging (Albany NY). 2018;10:3957-85. https:// doi.org/10.18632/aging.101685.
84. Li H, Bullock K, Gurjao C, et al. Metabolomic adaptations and correlates of survival to immune checkpoint blockade. Nat Commun. 2019;10:4346. https://doi.org/10.1038/ s41467-019-12361-9.

85. Netti GS, Lucarelli G, Spadaccino F, et al. PTX3 modulates the immunoflogosis in tumor microenvironment and is a prognostic factor for patients with clear cell renal cell carcinoma. Aging (Albany NY). 2020;12:7585-602. https://doi.org/10.18632/ aging.103169.

86. Tannir NM, Fan AC, Lee RJ, et al. Phase 1 study of glutaminase (GLS) inhibitor CB-839 combined with either everolimus (E) or cabozantinib (Cabo) in patients (pts) with clear cell (cc) and papillary (pap) metastatic renal cell cancer (mRCC). J Clin Oncol. 2018;36:603-603. https://doi.org/10.1200/JCO.2018.36.6_suppl. 603.

87. https://clinicaltrials.gov/ct2/show/NCT03463681. [Accessed on 2 April 2021.

88.• Graham J, Shah AY, Wells JC, et al. Outcomes of patients with metastatic renal cell carcinoma treated with targeted therapy after immuno-oncology checkpoint inhibitors. Eur Urol Oncol. 2021;4:102-11. https://doi.org/10.1016/j.euo.2019.11.001. (This retrospective study investigates the clinical outcome of targeted therapies after treatment with ICIs, and it is among the first analysis observing the effectiveness of sequential therapies for $\mathbf{m R C C}$ patients in real-world settings.)

89.• Auvray M, Auclin E, Barthelemy P, et al. Second-line targeted therapies after nivolumab-ipilimumab failure in metastatic renal cell carcinoma. Eur J Cancer. 2019;108:33-40. https://doi.org/10. 1016/j.ejca.2018.11.031. (This study reports the good outcome of these quential treatment with TKI after first-line combination nivolumab-ipilimumab in $\mathrm{mRCC}$, highlighting the need for trials investigating the optimal sequential treatment.)

90. Kuusk T, Albiges L, Escudier B, et al. Antiangiogenic therapy combined with immune checkpoint blockade in renal cancer. Angiogenesis. 2017;20:205-15. https://doi.org/10.1007/ s10456-017-9550-0.

91. Tumeh PC, Harview CL, Yearley JH, et al. PD-1 blockade induces responses by inhibiting adaptive immune resistance. Nature. 2014;515:568-71. https://doi.org/10.1038/nature13954.

92. Kim HJ, Cantor H. CD4 T-cell subsets and tumor immunity: the helpful and the not-so-helpful. Cancer Immunol Res. 2014;2:918. https://doi.org/10.1158/2326-6066.CIR-13-0216.

93. Escudier B, Sharma P, Mcdermott DF, et al. CheckMate 025 randomized phase 3 study: outcomes by key baseline factors and prior therapy for nivolumab versus everolimus in advanced renal cell carcinoma. Eur Urol. 2017;72:962-71. https://doi.org/ 10.1016/j.eururo.2017.02.010.

94. Chevrier S, Levine JH, Zanotelli VRT, et al. An immune atlas of clear cell renal cell carcinoma. Cell. 2017;169(736-749):e18. https://doi.org/10.1016/j.cell.2017.04.016.

95. Sharma P, Hu-Lieskovan S, Wargo JA, et al. Primary, adaptive, and acquired resistance to cancer immunotherapy. Cell. 2017;168:707-23. https://doi.org/10.1016/j.cell.2017.01.017.

96. Gopalakrishnan V, Spencer CN, Nezi L, et al. Gut microbiome modulates response to anti-PD-1 immunotherapy in melanoma patients. Science. 2018;359:97-103. https://doi.org/10.1126/ science.aan 4236.

97. Cianci R, Franza L, Schinzari G, et al., The interplay between immunity and microbiota at intestinal immunological niche: the case of cancer. Int J Mol Sci, 2019;20:https://doi.org/10. 3390/ijms20030501. (This review discusses the relationship between microbiota and immune niche in the intestinal mucosa and the possible impact on cancer onset, development, and response to treatment.)

98. Sender R, Fuchs S, Milo R. Revised estimates for the number of human and bacteria cells in the body. PLoS Biol. 
2016;14:e1002533. https://doi.org/10.1371/journal.pbio.10025 33.

99 Morgan XC, Huttenhower C. Chapter 12: Human microbiome analysis. PLoS Comput Biol. 2012;8:e1002808.

100. Levy M, Kolodziejczyk AA, Thaiss CA, et al. Dysbiosis and the immune system. Nat Rev Immunol. 2017;17:219-32. https://doi. org/10.1038/nri.2017.7.

101. Sivan A, Corrales L, Hubert N, et al. Commensal Bifidobacterium promotes antitumor immunity and facilitates anti-PD-L1 efficacy. Science. 2015;350:1084-9. https://doi.org/10.1126/ science.aac4255.

102. Vetizou M, Pitt JM, Daillere R, et al. Anticancer immunotherapy by CTLA- 4 blockade relies on the gut microbiota. Science. 2015;350:1079-84. https://doi.org/10.1126/science.aad1329.

103. Matson V, Fessler J, Bao R, et al. The commensal microbiome is associated with anti-PD-1 efficacy in metastatic melanoma patients. Science. 2018;359:104-8. https://doi.org/10.1126/scien ce.aao3290.

104. Routy B, Le Chatelier E, Derosa L, et al. Gut microbiome influences efficacy of PD-1-based immunotherapy against epithelial tumors. Science. 2018;359:91-7. https://doi.org/10.1126/scien ce.aan3706.

105. Frankel AE, Coughlin LA, Kim J, et al. Metagenomic shotgun sequencing and unbiased metabolomic profiling identify specific human gut microbiota and metabolites associated with immune checkpoint therapy efficacy in melanoma patients. Neoplasia. 2017;19:848-55. https://doi.org/10.1016/j.neo.2017.08.004.

106. Chaput N, Lepage P, Coutzac C, et al. Baseline gut microbiota predicts clinical response and colitis in metastatic melanoma patients treated with ipilimumab. Ann Oncol. 2017;28:1368-79. https://doi.org/10.1093/annonc/mdx108.

107. Ianiro G, Rossi E, Thomas AM, et al. Faecal microbiota transplantation for the treatment of diarrhoea induced by tyrosinekinase inhibitors in patients with metastatic renal cell carcinoma. Nat Commun. 2020;11:4333. https://doi.org/10.1038/ s41467-020-18127-y. (An innovative clinical trial showing the involvement of gut microbiota in the onset of diarrhea as an adverse event in TKI treatment of mRCC patients.)

108.•• Albiges L, Powles T, Staehler M, et al. Updated European Association of Urology Guidelines on renal cell carcinoma: immune checkpoint inhibition is the new backbone in first-line treatment of metastatic clear-cell renal cell carcinoma. Eur Urol. 2019;76:151-6. https://doi.org/10.1016/j.eururo.2019.05. 022. (This paper reports the update of the guidelines for the treatment of RCC patients by the European Association of Urology in light of the most recent trials with the combination therapies with ICIs.)

Publisher's Note Springer Nature remains neutral with regard to jurisdictional claims in published maps and institutional affiliations. 